\title{
The Development of a Dual-Radar System with Automatic Hypopnea Threshold Optimization for Contact-Free Sleep Apnea-Hypopnea Syndrome Screening
}

\author{
Shinji Gotoh $\mathbb{D}^{1},{ }^{1}$ Takemi Matsui $\mathbb{D}^{2}{ }^{2}$ Yoshikazu Naka, ${ }^{3}$ and Osamu Kurita ${ }^{3}$ \\ ${ }^{1}$ Department of Planning and Development, TAU GIKEN Co. Ltd., 814 Saedo-cho, Tsuzuki-ku, Yokohama, \\ Kanagawa 224-0054, Japan \\ ${ }^{2}$ Faculty of System Design, Tokyo Metropolitan University, 6-6 Asahigaoka, Hino, Tokyo 191-0065, Japan \\ ${ }^{3}$ Tomei Atsugi Hospital, 243-8571 232 Funako, Atsugi, Kanagawa, Japan
}

Correspondence should be addressed to Shinji Gotoh; s-gotoh@taugiken.jp

Received 29 August 2017; Accepted 10 December 2017; Published 8 January 2018

Academic Editor: Romeo Bernini

Copyright (c) 2018 Shinji Gotoh et al. This is an open access article distributed under the Creative Commons Attribution License, which permits unrestricted use, distribution, and reproduction in any medium, provided the original work is properly cited.

Full-night polysomnography (PSG) examination is regarded as the gold standard for the diagnosis of sleep apnea-hypopnea syndrome (SAHS). However, PSG requires the placement of multiple sensors on the head, face, and chest, which can impose a heavy strain on patients. Therefore, in the present study, we aimed to develop a contact-free, stand-alone SAHS screening system that eliminates body movement artifacts based on automatic optimization of the hypopnea threshold. Doppler radar sensors were placed beneath a mattress. In order to achieve high sensitivity and specificity, the hypopnea was based on the average amplitude of respiration during the full sleep period. The threshold was determined via receiver operating characteristic (ROC) analysis using PSG as a reference. We conducted full-night clinical tests of the proposed system in 27 patients with suspected SAHS ( $49 \pm 12$ years) at Tomei Atsugi Hospital. When predicting the severity of SAHS with an apnea-hypopnea index (AHI) of $>30 / h$ using PSG as a reference, the proposed system achieved a sensitivity of $100 \%$ and a specificity of $100 \%$. These results represent a drastic improvement over those of our previous study (sensitivity: $90 \%$; specificity: $79 \%$ ).

\section{Introduction}

Sleep disorders such as sleep apnea-hypopnea syndrome (SAHS) were first recognized as an important public health concern in the late 1970s [1]. Since its approval for clinical use, polysomnography (PSG) examination has been regarded as the gold standard for the diagnosis of SAHS. However, PSG requires the placement of multiple sensors on the head, face, and chest, which can impose a heavy strain on patients. To address these issues, early research focused on the development of clinically useful microwave radar (radar) systems for monitoring vital signs and body movements during sleep $[2,3]$. Although research regarding contactfree, radar-based respirometry and heart rate monitoring proved promising in the early 2000s, most studies at this time were laboratory investigations [4-6], and clinical studies did not begin until the first decade of the twenty-first century $[7,8]$. However, in the late 1990s, our research group began to investigate the use of Doppler radar-based respirometry for identifying survivors trapped beneath debris following massive earthquakes. Our findings indicated that this system, which utilizes antennae that can be inserted into heavy debris, exhibited high sensitivity for the identification of survivors $[9,10]$.

Based on these findings, we began to investigate the applicability of small microwave Doppler radar modules for the measurement of human vital signs $[7,11,12]$. In our recent studies, we compared the clinical usefulness of radar and $\mathrm{CO}_{2}$ sensors [13], radar and $\mathrm{SpO}_{2}$ sensors [14], and radar and PSG [15]. As the absolute values of respiration amplitude change depending on sleep state or changes in posture, we developed a method for detecting hypopnea based on relative decreases in amplitude every 30 seconds, without the use of a baseline value. Although this method yielded relatively good 
results [14], artifacts associated with body movement remained, resulting in low specificity. Several other research groups have also attempted to eliminate interference caused by body movement during sleep [16-18], with limited success. Also, Anishchenko et al. [19] proposed artifact removal method using Toeplitz matrix for rodents' respiratory activity in laboratory. Baboli et al. [20] presented radar monitoring system integrated with PSG system. Zhang et al. [21] proposed sleep stages classification using deep learning with bagged tree algorithm applied to laboratory experiments; participants were a couple of subjects. Beattie et al. [22] presented successful accurate scoring of AHI using load cells under the supports of bed. They achieved 100\% sensitivity and $97 \%$ specificity, at cutoff PSG AHI > 30. However, their classification was conducted by subjective assessment.

Although the use of additional biometric data may allow for the comprehensive elimination of noise based on the timing of body movements [16-18], no single system to date has achieved this aim. Therefore, in the present study, we aimed to develop a stand-alone screening system for the elimination of body movement artifacts based on automatic optimization of the hypopnea threshold. In this system, candidate events (i.e., relative decreases in amplitude) are filtered out based on an individual threshold derived from full sleep-span data.

\section{Materials and Methods}

2.1. Configuration of the System. To overcome the effects of posture change during sleep, we utilized two radar systems to ensure that respiration was always detected by at least one sensor (MVM, TAU GIKEN Co. Ltd. Yokohama, Japan; Doppler module NJR 4169J, New Japan Radio Co. Ltd., Tokyo, Japan). The system configuration is depicted in Figure 1(a). The system specifications were as follows: (a) emitting frequency of $10.525 \mathrm{GHz}$, (b) emitting power of approximately $10 \mathrm{~mW}$, and (c) antenna gain of $5 \mathrm{dBi}$. Using quadrature direct conversion technique [23, 24], received signals were converted into in-phase output and quadrature output. Then, these outputs were filtered by analog band-pass filters, where adjusting processes to balance amplitude of both output and to eliminate phase noise were also conducted. The two radar sensors were located beneath the mattress, $20 \mathrm{~cm}$ from the left and right of the midline of the participant's body, near the iliac bone (Figure 1(b)). Data from the two radar systems were integrated following measurement.

The $10.525 \mathrm{GHz}$ radar output was filtered and digitized in the main unit at a rate of 20 times/s. The data were transferred to a personal computer via wireless LAN and stored as CSV files (Figure 1(c)). Following data collection, offline calculations were performed in Microsoft Excel to identify signs of respiratory disorders. The stored files consisted of the formatted data with time stamps every $0.05 \mathrm{~s}$. The total number of data points was approximately 576,000 (i.e., 20 times $\left.\mathrm{s}^{-1} \times 60 \mathrm{~s} \times 60 \mathrm{~m} \times 8 \mathrm{~h}\right)$.

2.2. Automatic Optimization of Hypopnea Threshold. In order to achieve high sensitivity and specificity for the detection of SAHS, we developed a novel tool for automatic optimization of the hypopnea threshold. Data were analyzed using Microsoft Excel, in accordance with the following protocol: The quadrature detector output values $E_{\mathrm{I}}$ and $E_{\mathrm{Q}}$ ( $E_{\mathrm{I}}=$ in-phase output, $E_{\mathrm{Q}}=$ quadrature output) were used to calculate the phase deviation of the received signal. $E_{\mathrm{I}}$ and $E_{\mathrm{Q}}$ values were obtained for each stored data point. Using $E_{\mathrm{I}}$ and $E_{\mathrm{Q}}$, the phase of respiration was calculated according to (1), where $\theta(t)$ (degrees) represents the phase at time $t$ (s) and atan represents the arctangent in radians:

$$
\theta(t)=\operatorname{atan}\left(\frac{E_{\mathrm{Q}}}{E_{\mathrm{I}}}\right) \cdot \frac{180}{\pi} .
$$

Amplifiers and filters were driven by a single $5 \mathrm{~V}$ DC power source. A center value of $2.5 \mathrm{~V}$ was used for output signals, resulting in periodic vibration of $\theta(t)$ at a center value of 45 degrees. Then, the magnitude of respiration over $5 \mathrm{~s}(m(t)$ in degrees) was calculated according to (2), where $\Delta t=0.05 \mathrm{~s}$ :

$$
\begin{aligned}
m(t)=\{ & \max [\theta(t), \theta(t+\Delta t), \ldots, \theta(t+5)] \\
& -\min [\theta(t), \theta(t+\Delta t), \ldots, \theta(t+5)]\} .
\end{aligned}
$$

The functions $\max []$ and $\min []$ were used to extract the maximum and minimum values, respectively.

Using three $m(t)$ values, $M(t)$ and $M(t-15)$ were calculated in degrees as follows:

$$
\begin{aligned}
M(t) & =\frac{m(t)+m(t+5)+m(t+10)}{3} \\
M(t-5) & =\frac{m(t-15)+m(t-10)+m(t-5)}{3} .
\end{aligned}
$$

Then, the event markers $E(t)$ were calculated as follows:

$$
\begin{aligned}
& \mathrm{IF} \frac{M(t-15)}{M(t)} \geq 2 \operatorname{AND}\{\max [m(t), m(t+5), m(t+10), m \\
& \cdot(t-5), m(t-10), m(t-15)]<\mu\}, \\
& E(t)=1, \text { else } E(t)=0,
\end{aligned}
$$

where $\mu$ was calculated as

$$
\mu=K \cdot \frac{1}{L} \cdot \sum_{n=0}^{L-1} m(0+n \cdot \Delta t) .
$$

As shown in (5), $\mu$ represented average amplitude of overall sleeping time.

$K$ was determined to optimize sensitivity and specificity via receiver operating characteristic (ROC) analysis based on SAHS patients' data using PSG as a reference. $L$ represented the approximate number of data points $(576,000)$, and $\Delta t=0.05 \mathrm{~s}$. The $E(t)$ was calculated every 30 seconds and stored in the data file, and the consecutive portions of $E(t)=1$ were counted as a single event. Separate $E(t)$ values were calculated for radar1 and radar2 $\left(E(t)_{\text {radar1 }}\right.$ and $E(t)_{\text {radar2 }}$, resp.). To integrate the data from the two radar 


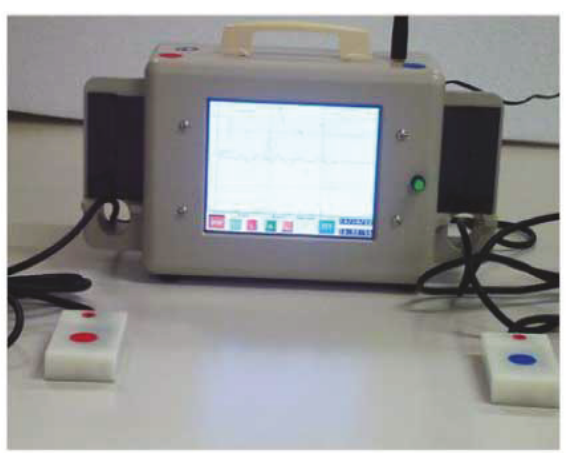

(a)

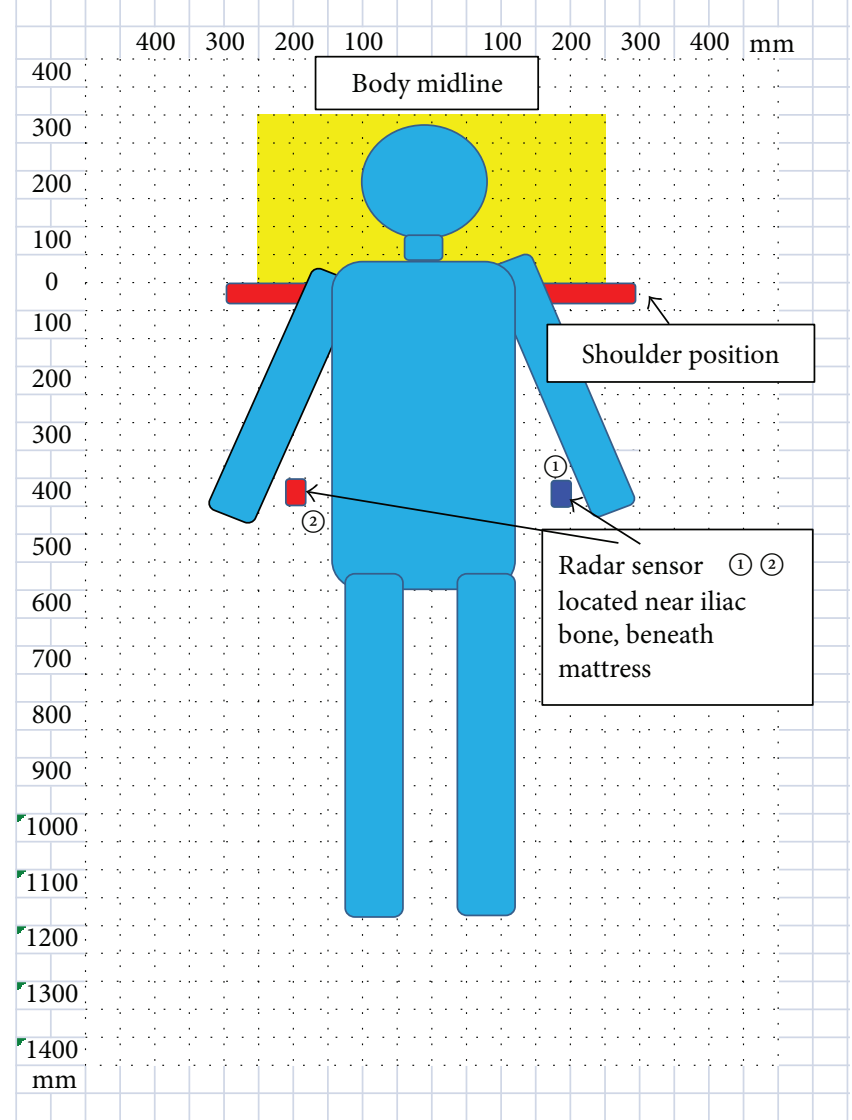

(b)

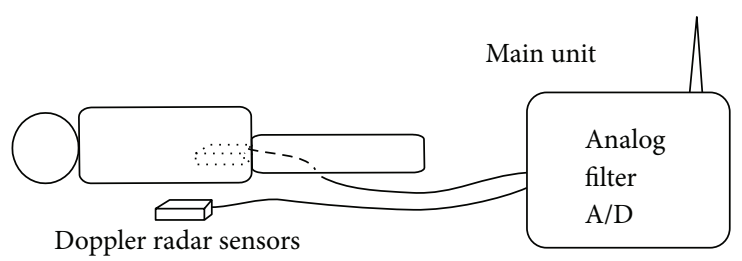

Doppler radar sensors

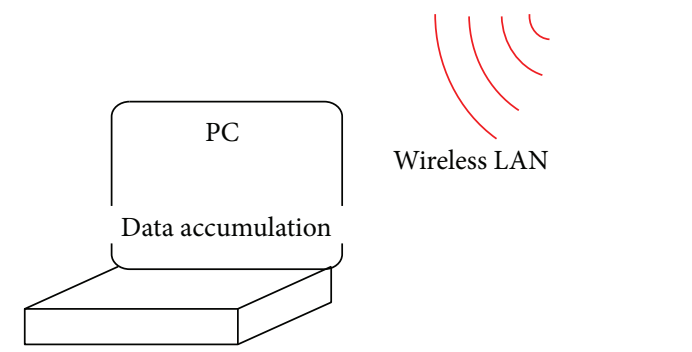

(c)

Figure 1: The dual Doppler radar system. (a) A picture of the main unit and sensors. (b) The sensors were located $20 \mathrm{~cm}$ to the left and right of the patient's midline, near the iliac bone. (c) Schematic of respiration measurement.

systems, the logical sum of these values was then calculated in Excel as follows:

$$
E(t)_{\text {merged }}=E(t)_{\text {radar1 }} \cup E(t)_{\text {radar2 }}
$$

Finally, $E(t)_{\text {merged }}$ values of 1 were considered indicative of respiratory disorder.
A value of $(M(t-15) / M(t)) \geq 2$ was considered indicative of hypopnea based on the $\mathrm{AHI}_{\text {Chicago }}$ criteria provided by the American Academy of Sleep Medicine (AASM), in which hypopnea is indicated by $>50 \%$ reduction in air flow for more than $10 \mathrm{~s},>3 \%$ oxygen desaturation, or arousal. Although radar measurements of body surface area are associated with airflow, they do not exhibit one-to-one 
TABLE 1: Number of radar detected hypopnea events.

\begin{tabular}{|c|c|c|c|c|c|c|c|c|c|c|c|}
\hline \multirow[t]{2}{*}{ Participant } & \multirow[t]{2}{*}{ AHI by PSG } & \multicolumn{2}{|c|}{$\begin{array}{l}\text { Average } \\
\text { amplitude } \\
\text { (degree) }\end{array}$} & \multicolumn{7}{|c|}{ Number of radar detected events corresponding to $K$ value } & \multirow[t]{2}{*}{ Before optimizing } \\
\hline & & Radar1 & Radar2 & $K=0.25$ & $K=0.5$ & $K=0.75$ & $K=1.0$ & $K=1.25$ & $K=1.5$ & $K=1.75$ & \\
\hline 1 & 8.3 & 5.8 & 4.8 & 4 & 17 & 48 & 65 & 75 & 92 & 108 & 161 \\
\hline 2 & 12.0 & 7.9 & 7.1 & 25 & 46 & 62 & 74 & 76 & 80 & 83 & 102 \\
\hline 3 & 14.0 & 6.3 & 5.6 & 22 & 56 & 80 & 100 & 120 & 137 & 155 & 246 \\
\hline 4 & 15.6 & 4.5 & 4.9 & 10 & 43 & 65 & 85 & 106 & 121 & 129 & 144 \\
\hline 5 & 15.6 & 4.2 & 2.5 & 24 & 62 & 99 & 122 & 138 & 156 & 167 & 211 \\
\hline 6 & 19.2 & 3.3 & 5.3 & 16 & 49 & 82 & 107 & 124 & 145 & 149 & 174 \\
\hline 7 & 20.0 & 8.2 & 5.9 & 23 & 62 & 90 & 118 & 131 & 163 & 172 & 191 \\
\hline 8 & 20.2 & 2.9 & 2.4 & 6 & 29 & 71 & 112 & 141 & 152 & 161 & 173 \\
\hline 9 & 21.8 & 4.8 & 4.8 & 26 & 69 & 95 & 111 & 136 & 163 & 189 & 259 \\
\hline 10 & 26.0 & 4.8 & 2.7 & 21 & 57 & 79 & 90 & 104 & 118 & 122 & 160 \\
\hline 11 & 26.0 & 3.3 & 3.8 & 7 & 60 & 105 & 159 & 198 & 240 & 260 & 358 \\
\hline 12 & 28.5 & 4.7 & 3.8 & 21 & 61 & 88 & 114 & 140 & 160 & 170 & 184 \\
\hline 13 & 29.2 & 3.5 & 3.5 & 49 & 105 & 142 & 160 & 182 & 192 & 204 & 236 \\
\hline 14 & 29.9 & 3.0 & 2.5 & 6 & 55 & 105 & 137 & 158 & 175 & 189 & 194 \\
\hline 15 & 37.7 & 7.0 & 6.7 & 60 & 126 & 169 & 199 & 233 & 264 & 285 & 301 \\
\hline 16 & 39.2 & 7.5 & 6.2 & 76 & 257 & 399 & 501 & 567 & 612 & 651 & 744 \\
\hline 17 & 39.6 & 16.2 & 19.1 & 54 & 152 & 235 & 320 & 383 & 421 & 460 & 466 \\
\hline 18 & 39.6 & 10.9 & 12.9 & 113 & 296 & 424 & 512 & 575 & 619 & 663 & 673 \\
\hline 19 & 43.2 & 7.9 & 3.9 & 14 & 57 & 114 & 162 & 190 & 207 & 221 & 276 \\
\hline 20 & 47.6 & 5.0 & 11.7 & 63 & 173 & 268 & 335 & 377 & 431 & 455 & 497 \\
\hline 21 & 59.5 & 6.3 & 5.9 & 28 & 85 & 162 & 234 & 284 & 321 & 349 & 398 \\
\hline 22 & 59.5 & 5.1 & 8.3 & 56 & 148 & 221 & 269 & 308 & 333 & 350 & 386 \\
\hline 23 & 64.0 & 3.9 & 4.0 & 65 & 197 & 314 & 358 & 381 & 389 & 405 & 414 \\
\hline 24 & 67.5 & 12.4 & 17.5 & 94 & 220 & 313 & 416 & 500 & 555 & 615 & 584 \\
\hline 25 & 68.1 & 9.0 & 11.6 & 33 & 117 & 161 & 189 & 213 & 233 & 244 & 212 \\
\hline 26 & 71.4 & 13.0 & 9.7 & 178 & 402 & 562 & 687 & 761 & 828 & 878 & 936 \\
\hline 27 & 99.2 & 12.1 & 11.0 & 136 & 266 & 368 & 456 & 503 & 537 & 559 & 595 \\
\hline
\end{tabular}

Column 2: individual apnea-hypopnea index obtained via PSG. Columns 3 and 4: the average radar-detected respiration amplitude. Columns 5 to 11: number of radar-detected events corresponding to $K$ value. Column 12: radar detected events before automatic normal-hypopnea threshold optimization.

correspondence with airflow due to changes in the distance/angle between the radar sensors and the body. Thus, we were unable to utilize the revised AASM 2012 criteria, which specify apnea and hypopnea threshold values of $90 \%$ and $30 \%$ decreases in amplitude relative to baseline, respectively. Although these criteria may be applicable when patients do not change posture or for measurement during a single stage of sleep, we considered these criteria too stringent for radar-based measurement. Therefore, simplified criteria were adopted to identify cases of probably sleep-disordered breathing in the present and previous studies $[14,15]$.

Thus, the optimizer first identified candidates for respiratory disorder, following which it evaluated these candidates based on $K$ and $\mu$.

2.3. Clinical Testing and $K$ Value Optimization. We conducted clinical tests of the proposed system in 27 patients (mean age: $49 \pm 12$ years; 22 men, 5 women) hospitalized one night for the evaluation of suspected SAHS from May to September of 2014 and February to March of 2015. All patients provided written informed consent to participate in the present study. Patients underwent simultaneous evaluation via PSG (Sleep Watcher E-series, Compumedics Ltd., Victoria, Australia; data processing: Teijin Pharma Ltd., Tokyo, Japan) and radar-based screening. The PSG determines AHIs based on AASM 2012 criteria [25]. The duration of measurement was $8 \mathrm{~h}$ in all cases. This study was approved by the Ethics Committee of Tomei Atsugi Hospital (Atsugi, Kanagawa, Japan).

Receiver operating characteristic (ROC) analysis was conducted to determine the appropriate $K$ value. $K$ values were increased from 0.25 to 1.75 in increments of 0.25 , and the number of derived $E(t)_{\text {merged }}$ events was calculated at each step. The results of this process are summarized in Table 1. PSG-based diagnoses were used in conjunction with $\mathrm{R}$ version 3.3.3. and ROCR 1.0-5 library to select the conditions most appropriate for determining an $\mathrm{AHI} \geq 30 / \mathrm{h}$. A $K$ 


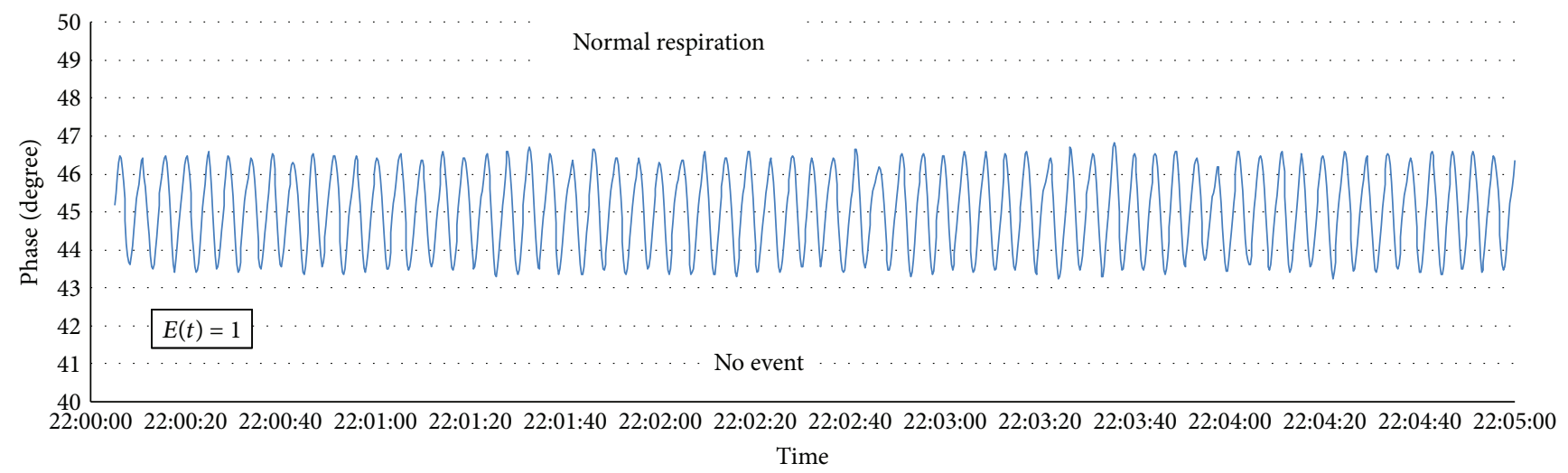

FIgURE 2: Typical sample of normal respiration. AHI $=14$.
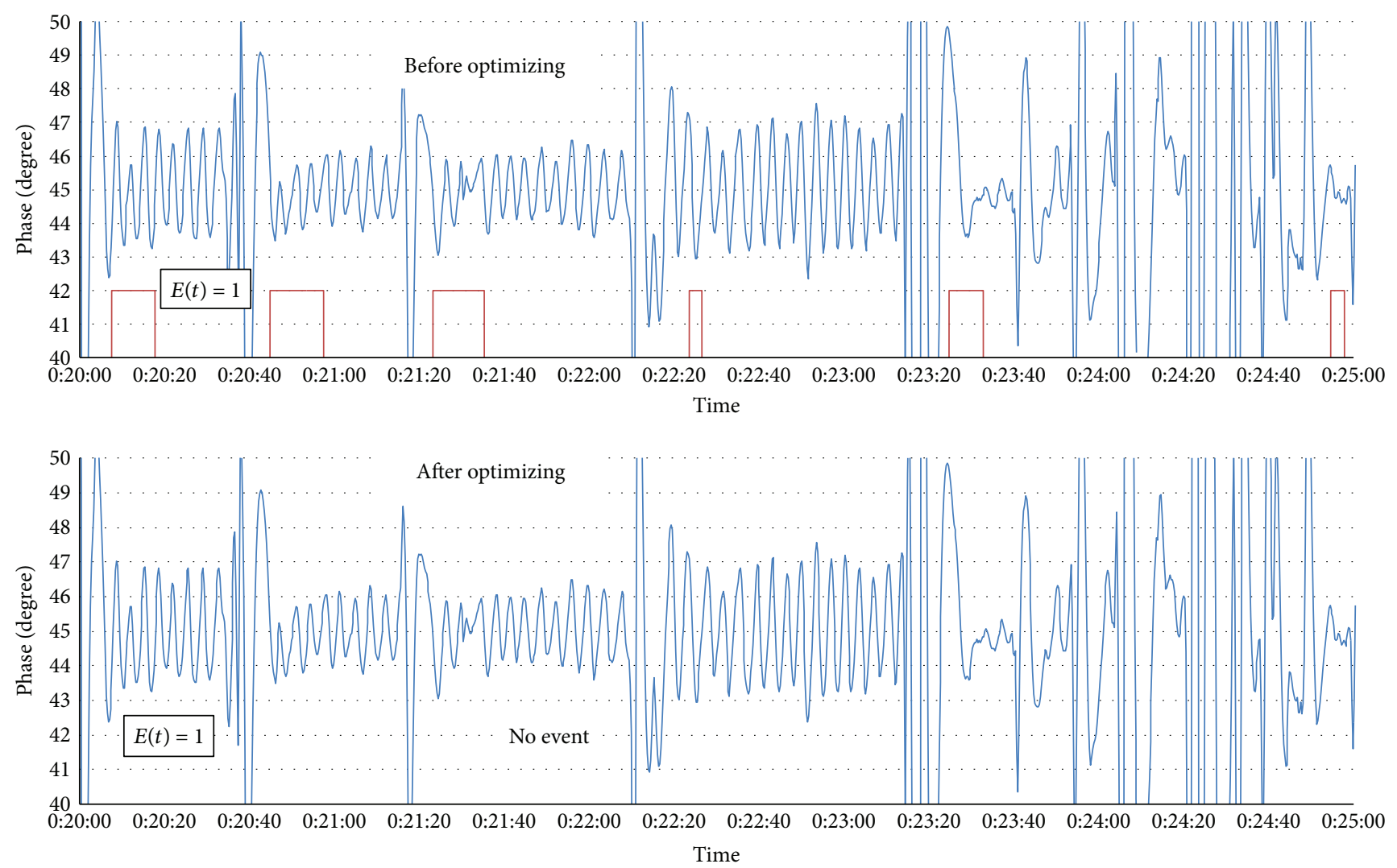

Figure 3: Typical sample of body movement noises, before optimizing and after optimizing. Body-movement-like noises are eliminated. $\mathrm{AHI}=14$ participants.

value of 1.0 yielded the best results. The optimized $K$ value was incorporated into the software used for analysis of patient data. During measurement, the hypopnea threshold is automatically updated by the minute to achieve high sensitivity and specificity.

\section{Results}

A representative data sample from a patient with normal respiration is presented in Figure 2. In this case, no hypopnea events have been identified, and $E(t)$ is always equal to 0 . Figure 3 includes data from a patient with strong body movement both prior to and following optimization. Figure 4 presents pre- and postoptimization data from a patient exhibiting both hypopnea and body movement $(\mathrm{AHI}=14)$. As shown in Figures 3 and 4, the automatic hypopnea threshold optimizer effectively reduced noise associated with body movement: Figure 3 depicts the elimination of six events associated with body movement. As there were no events during this period, $E(t)$ was always equal to 0 . In Figure 4, 

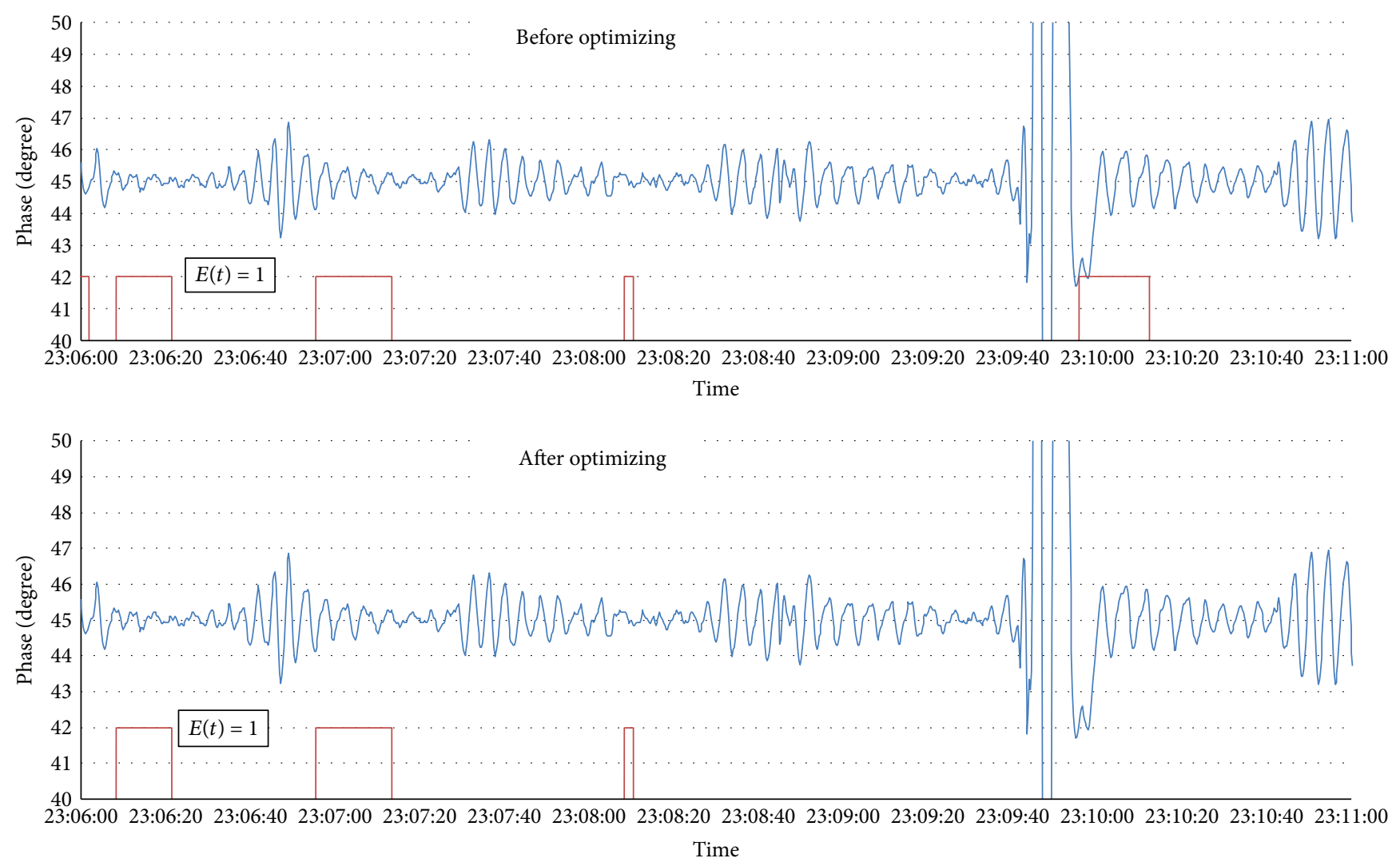

FIGURE 4: Hypopnea and body-movement noise in a single patient. After optimization, only body-movement-like events have been eliminated. $\mathrm{AHI}=14$.

frequent hypopnea events and one large deviation due to body movement can be observed, resulting in the generation of four events. Following optimization, noise associated with the body movement event had been eliminated, although the three hypopnea events remained. These findings indicate that the automatic hypopnea threshold optimizer effectively eliminated noise associated with body movement. The individual mean amplitude values for all participants are summarized in Table 1. Mean values were calculated separately for each radar system. As shown in Table 1, the numbers of radar detected events unproportionally increased along with $K$ value. Average radar amplitudes of dual radars were weakly correlated with AHIs $(r=0.51$ $(\operatorname{radar} 1), r=0.52(\operatorname{radar} 2))$.

ROC analysis was utilized to determine the appropriate $K$ value prior to clinical testing. Only $K=1.0$ yielded sensitivity and specificity values of $100 \%$. The results of ROC analysis before and after optimization are presented in Figures 5(a)5(d). Figures 5(a) and 5(c) represent the analysis using an AHI cutoff of 30, while Figures 5(b) and 5(d) represent the analysis using an AHI cutoff of 15.

When $K=1.0$ and predictions of SAHS severity were based on an AHI $>30 / \mathrm{h}$ on PSG, the proposed system achieved a sensitivity of $100 \%$ and a specificity of $100 \%$. Under the same conditions without optimization, sensitivity and specificity were $92 \%$ and $93 \%$, respectively. Our findings further revealed that the number of events detected via radar can be approximated by multiplying the number of apnea- hypopnea events per hour by two-thirds. Thus, an AHI > 30/ $\mathrm{h}$ is equivalent to $>20$ radar events per hour.

\section{Discussion}

In the present study, we aimed to develop a stand-alone screening system for the elimination of body movement artifacts based on automatic optimization of the apneahypopnea threshold. Notably, we calculated the average amplitude of respiration for the full duration of sleep, while previous studies have utilized the mean value obtained from a small sample of sleep data in which respiration remained stable. However, this method of determining the individual patient's baseline value is problematic, as the resulting values cannot account for changes in sleep stage, arousal, and posture. Kagawa et al. [16] also used an amplitude-based detection method; they utilized $40 \mathrm{~s}$ of normal-amplitude data to determine the individual baseline, setting the thresholds for apnea and hypopnea to $20 \%$ and $70 \%$, of baseline, respectively. Using this method, Kagawa et al. achieved sensitivity and specificity values of $90 \%$ and $79 \%$, respectively, for predicting the severity of SAHS based on an apnea-hypopnea index (AHI) of $>30 / \mathrm{h}$. However, our automatic optimization method yielded much higher sensitivity and specificity of $100 \%$ and $100 \%$, respectively.

Our findings indicate that more stable measurements can be obtained by calculating the mean for the total duration of sleep. 


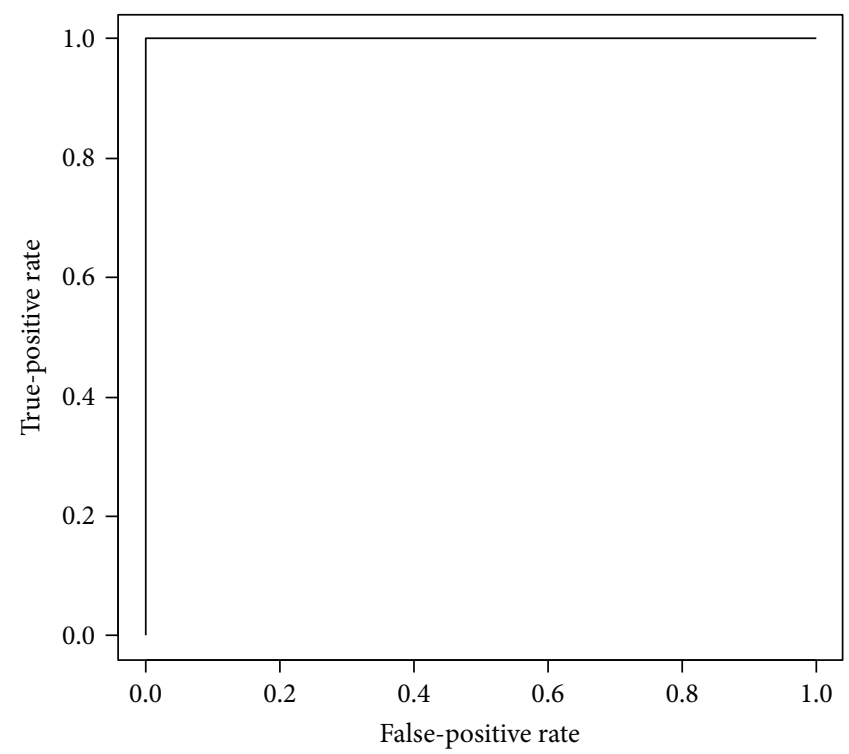

(a)

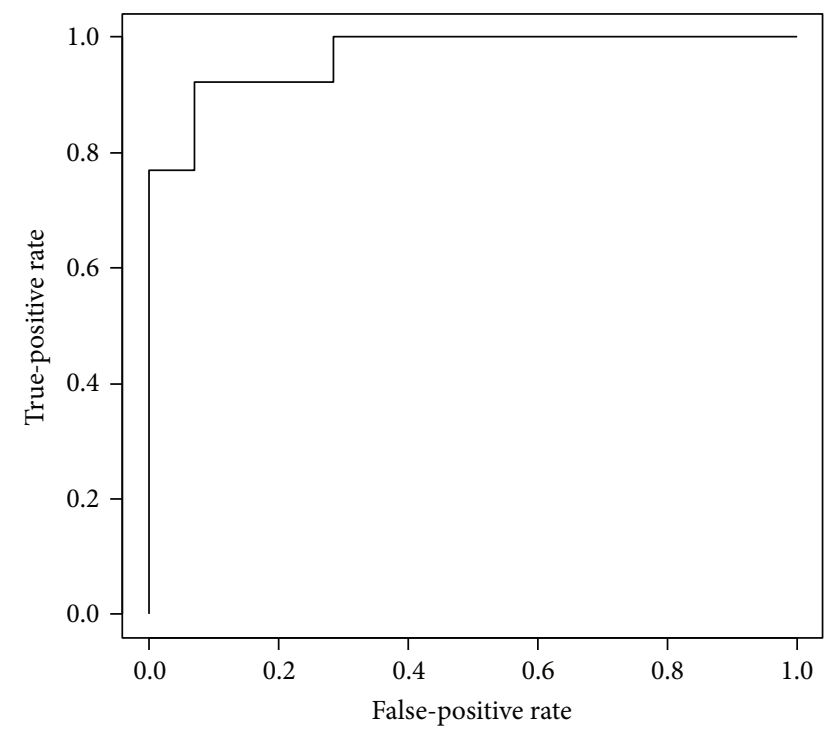

(c)

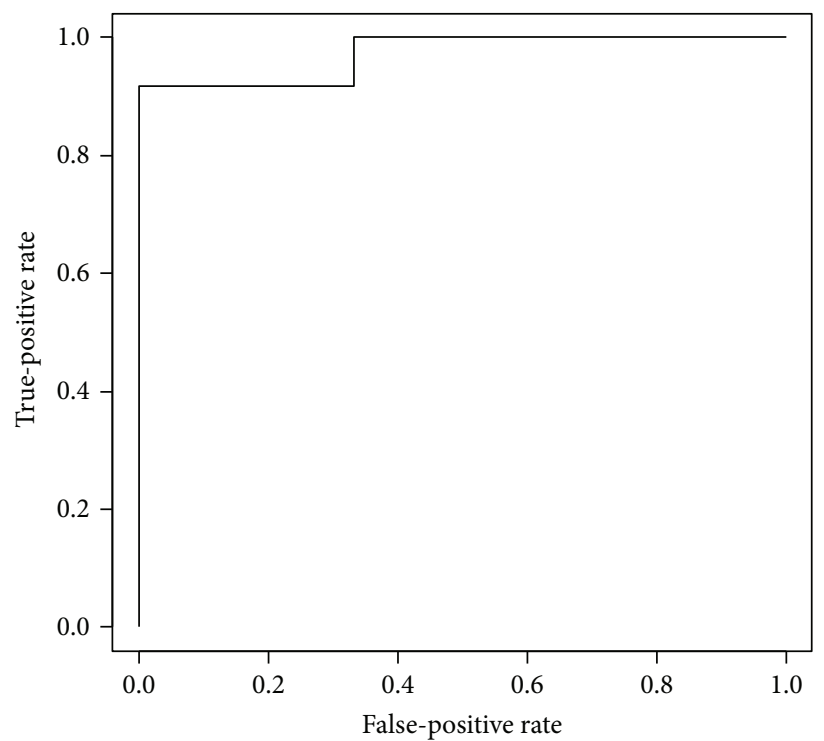

(b)

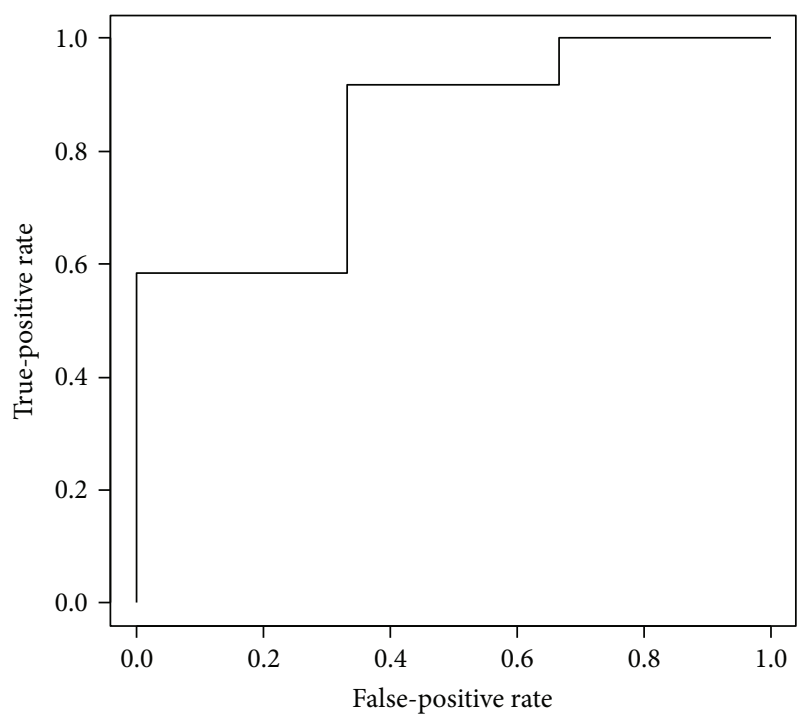

(d)

Figure 5: ROC curve $(K=1.0)$. Panel (a) presents an AHI $>30$ positive case, after optimization. Panel (b) presents an AHI $>15$ positive case, after optimization. Panel (c) presents an AHI $>30$ positive case, before optimization. Panel (d) presents an AHI $>15$ positive case, before optimization. All charts were made using R software.

Furthermore, we determined the optimum $K$ value via ROC analysis. Although the appropriate $K$ value was lower than expected, our findings indicated that the proposed method was effective in the screening and diagnosis of patients with SAHS. Our findings suggest that respiration data exhibit relatively small changes in overall amplitude and that interference caused by body movement produces large spikes in amplitude, which can be eliminated using a small $K$ value of 1 . However, as the present study included a small number of participants, further studies involving larger patient samples are required to determine the mechanisms underlying this effect. Furthermore, all participants of the present study had been diagnosed with SAHS. As such, future studies should include a control group of healthy participants to improve the generalizability of our findings.

\section{Conclusion}

The results of the present study indicate that measurement of respiration during sleep can be accomplished using radar-based systems equipped with an automatic hypopnea threshold optimizer. Moreover, our findings indicate that such noninvasive, stand-alone, and contact-free measurement can be used to screen for SAHS in patient homes and in more varied clinical settings. Such devices may reduce the incidence of serious complications associated with SAHS 
and other sleep disorder and reduce medical costs due to misdiagnosis of sleep disorders.

\section{Conflicts of Interest}

The authors declare that there are no conflicts of interest regarding the publication of this article.

\section{Acknowledgments}

This study was supported in part by the 2013-2015 "Growth, Industry, Innovation" project of the Kanagawa Prefectural Government.

\section{References}

[1] T. Young, M. Palta, J. Dempsey, J. Skatrud, S. Weber, and S. Badr, "The occurrence of sleep-disordered breathing among middle-aged adults," The New England Journal of Medicine, vol. 328, no. 17, pp. 1230-1235, 1993.

[2] J. C. Lin, "Microwave sensing of physiological movement and volume change: a review," BioElectroMagnetics, vol. 13, no. 6, pp. 557-565, 1992.

[3] I. Arai, K. Motomura, and T. Suzuki, "A microwave Doppler radar system for noncontact measurement of head and finger movements in clinical use," IEICE Transactions on Communications, vol. E76B, no. 10, pp. 1318-1324, 1993.

[4] D. Dei, G. Grazzini, G. Luzi et al., "Noncontact detection of breathing using a microwave sensor," Sensors, vol. 9, no. 4, pp. 2574-2585, 2009.

[5] W. Massagram, V. M. Lubecke, and O. Boric-Lubecke, "Microwave non-invasive sensing of respiratory tidal volume," in 2009 Annual International Conference of the IEEE Engineering in Medicine and Biology Society, pp. 4832-4835, Minneapolis, MN, USA, 2009.

[6] J.-H. Oum, D.-W. Kim, and S. Hong, "Two frequency radar sensor for non-contact vital signal monitor," in 2008 IEEE MTT-S International Microwave Symposium Digest, pp. 919922, Atlanta, GA, USA, USA, 2008.

[7] T. Matsui, S. Gotoh, I. Arai et al., "Noncontact vital sign monitoring system for isolation unit (casualty care system)," Military Medicine, vol. 171, no. 7, pp. 639-643, 2006.

[8] A. D. Droitcour, T. B. Seto, B. Park et al., "Non-contact respiratory rate measurement validation for hospitalized patients," in 2009 Annual International Conference of the IEEE Engineering in Medicine and Biology Society, pp. 4812-4815, Minneapolis, MN, USA, 2009.

[9] I. Arai, "Survivor search radar system for persons trapped under earthquake rubble," in APMC 2001. 2001 Asia-Pacific Microwave Conference (Cat. No.01TH8577), pp. 663-668, Taipei, Taiwan, Taiwan, 2001.

[10] “Japan Patent Office; patent publication," JP 3700-00954A, 2005.

[11] S. Suzuki, T. Matsui, H. Kawahara et al., "A non-contact vital sign monitoring system for ambulances using dual-frequency microwave radars," Medical \& Biological Engineering \& Computing, vol. 47, no. 1, pp. 101-105, 2009.

[12] S. Gotoh, T. Matsui, H. Imuta, M. Kagawa, Z. Badarch, and T. Matsui, "Non-contact determination of parasympathetic activation induced by a full stomach using microwave radar," Medical \& Biological Engineering \& Computing, vol. 47, no. 9, pp. 1017-1019, 2009.
[13] S. Gotoh, T. Matsui, K. Yamashita et al., "A study for novel non-contact sleep respiration disorder measuring system utilizing micro-wave radar and application to screening of sleep apnea syndrome and other sleep respiratory disorder," Iryou kikigaku (The Japanese journal of medical instrumentation), vol. 84, no. 3, pp. 317-324, 2014.

[14] S. Gotoh, T. Matsui, M. Kojima, K. Yamashita, and Y. Sakamoto, "Comparison study between micro wave radar and conventional $\mathrm{SpO}_{2}$ measurement for primary screening of sleep apnea syndrome," Journal of Life Support Engineering, vol. 26, no. 4, pp. 126-131, 2014.

[15] S. Gotoh, T. Matsui, K. Yamashita, H. Kaneko, Y. Naka, and O. Kurita, "A clinical study for non-contact sleep respirometry with microwave radar, monitored by simultaneous measuring with polysomnography," Iryou kikigaku (The Japanese journal of medical instrumentation), vol. 86, no. 5, pp. 441-449, 2016.

[16] M. Kagawa, H. Tojima, and T. Matsui, "Non-contact diagnostic system for sleep apnea-hypopnea syndrome based on amplitude and phase analysis of thoracic and abdominal Doppler radars," Medical \& Biological Engineering \& Computing, vol. 54, no. 5, pp. 789-798, 2016.

[17] M. Kagawa, Y. Yoshida, M. Kubota, A. Kurita, and T. Matsui, "Non-contact heart rate monitoring method for elderly people in bed with random body motions using $24 \mathrm{GHz}$ dual radars located beneath the mattress in clinical settings," Journal of Medical Engineering \& Technology, vol. 36, no. 7, pp. 344350, 2012.

[18] M. Kagawa, K. Ueki, H. Tojima, and T. Matsui, "Noncontact screening system with two microwave radars for the diagnosis of sleep apnea-hypopnea syndrome," in 201335 th Annual International Conference of the IEEE Engineering in Medicine and Biology Society (EMBC), pp. 2052-2055, Osaka, Japan, 2013.

[19] L. Anishchenko, G. Gennarelli, A. Tataraidze, E. Gaysina, F. Soldovieri, and S. Ivashov, "Evaluation of rodents' respiratory activity using a bioradar," IET Radar, Sonar \& Navigation, vol. 9, no. 9, pp. 1296-1302, 2015.

[20] M. Baboli, A. Singh, B. Soll, O. Boric-Lubecke, and V. M. Lubecke, "Good night: sleep monitoring using a physiological radar monitoring system integrated with a polysomnography system," IEEE Microwave Magazine, vol. 16, no. 6, pp. 3441, 2015.

[21] L. Zhang, J. Xiong, H. Zhao, H. Hong, X. Zhu, and C. Li, "Sleep stages classification by CW Doppler radar using bagged trees algorithm," in 2017 IEEE Radar Conference (RadarConf), pp. 0788-0791, Seattle, WA, USA, 2017.

[22] Z. T. Beattie, T. L. Hayes, C. Guilleminault, and C. C. Hagen, "Accurate scoring of the apnea-hypopnea index using a simple non-contact breathing sensor," Journal of Sleep Research, vol. 22, no. 3, pp. 356-362, 2013.

[23] B. Razavi, "Design consideration for direct-conversion receivers," IEEE Transactions on Circuits and Systems II: Analog and Digital Signal Processing, vol. 44, no. 6, pp. 428-435, 1997.

[24] G. Gennarelli, F. Soldovieri, L. Marciano, G. Cerasuolo, and O. Petrella, "Measurements performance of a bioradar for human respiration monitoring," Procedia Engineering, vol. 168, pp. 1200-1203, 2016.

[25] R. B. Berry, R. Budhiraja, D. J. Gottlieb et al., "Rules for scoring respiratory events in sleep: update of the 2007 AASM manual for the scoring of sleep and associated events. Deliberations of the sleep apnea definitions task force of the American Academy of Sleep Medicine," Journal of Clinical Sleep Medicine, vol. 8, no. 5, pp. 597-619, 2012. 


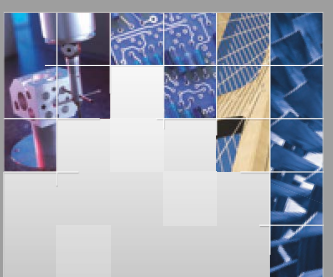

\section{Enfincering}
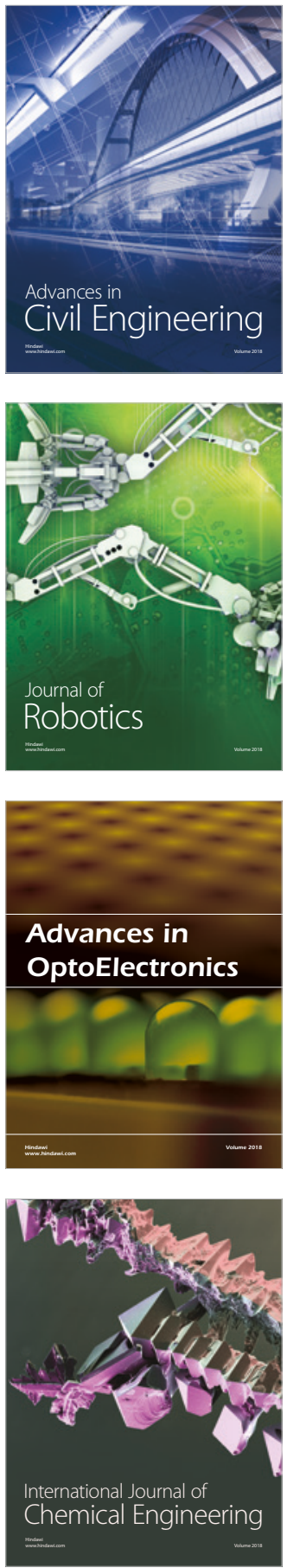

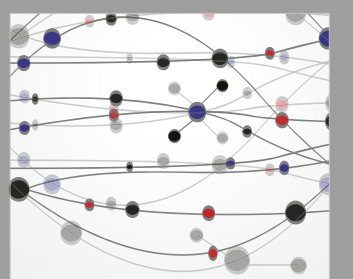

\section{Rotating \\ Machinery}

The Scientific World Journal

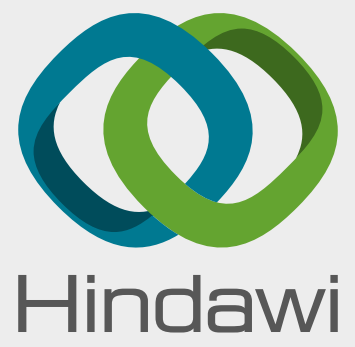

Submit your manuscripts at

www.hindawi.com
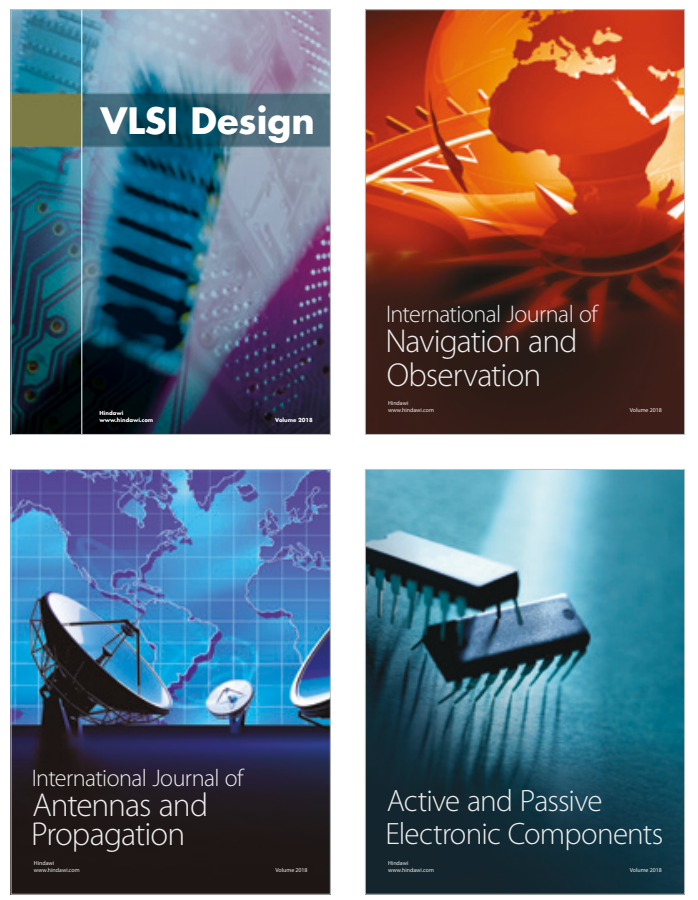
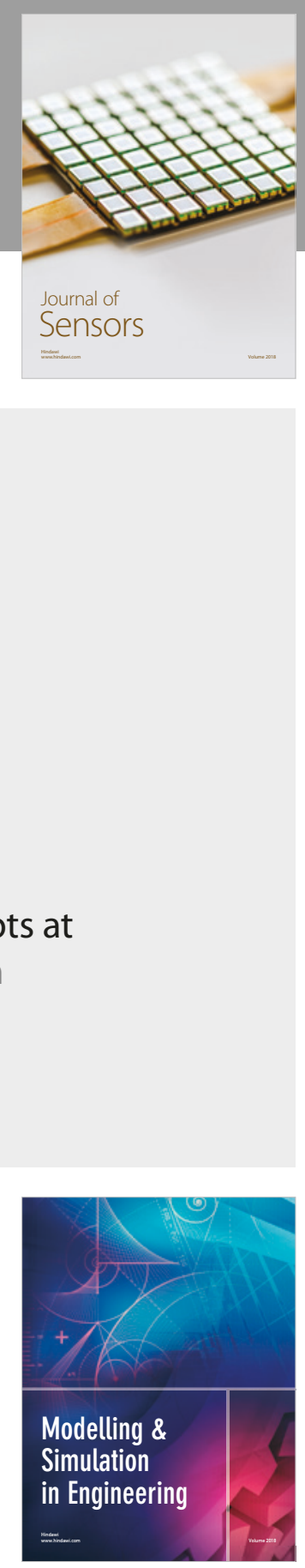

\section{Advances \\ Multimedia}
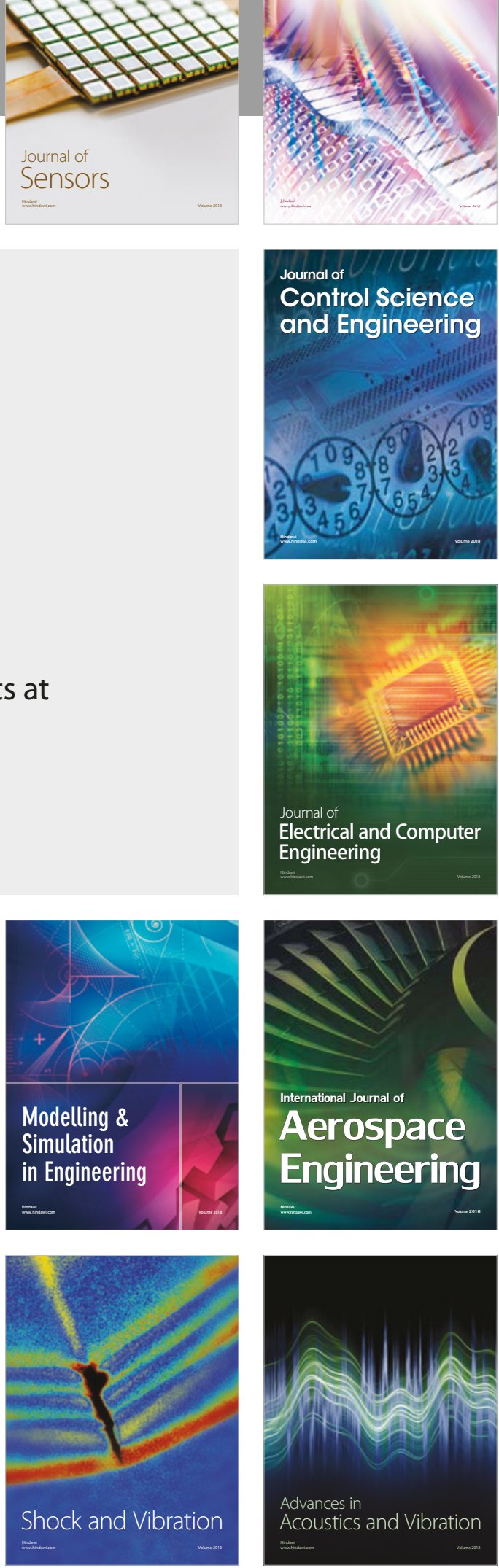